DFPD-12-TH-01

QMUL-PH-12-02

\title{
ADDING NEW HAIR TO THE 3-CHARGE BLACK RING
}

\author{
Stefano Giusto ${ }^{1,2}$, Rodolfo Russo ${ }^{3}$ \\ ${ }^{1}$ Dipartimento di Fisica "Galileo Galilei", \\ Università di Padova, \\ Via Marzolo 8, 35131 Padova, Italy \\ 2 INFN, Sezione di Padova, \\ Via Marzolo 8, 35131, Padova, Italy \\ ${ }^{3}$ Queen Mary University of London, \\ Centre for Research in String Theory, School of Physics \\ Mile End Road, London E1 4NS, UK \\ stefano.giusto@pd.infn.it, r.russo@qmul.ac.uk
}

\begin{abstract}
Motivated by the string theory analysis of [1], we construct a class of 1/8-BPS solutions of type IIB supergravity compactified on $S^{1} \times T^{4}$. In this duality frame our ansatz allows for a non-trivial NS-NS B-field which has been usually set to zero in previous studies of $1 / 8$-BPS geometries. We provide a M-theory description of these new geometries and show that they can be interpreted as the lift of solutions of the $\mathcal{N}=25 \mathrm{D}$ supergravity with three vector multiplets and whose scalar manifold is the symmetric space $S O(1,1) \otimes(S O(1,2) / S O(2))$. Finally we show that the non-minimal 5D black rings provide an explicit example of solutions falling in this ansatz. In particular we point out the existence of a black ring that has an extra dipole charge with respect to the solutions of the STU-model. In the near-horizon limit, this ring has an $\mathrm{AdS}_{3} \times S^{3}$ geometry with the same radius as the one of the 3-charge black hole and thus its microstates should belong to the usual D1-D5 CFT.
\end{abstract}




\section{Introduction}

D-brane configurations in type II string theories can be described both from a gravitational and a microscopic (i.e. conformal field theory) point of view, see for instance $[2,3]$ for reviews focusing on the first and the second aspect respectively. A link between these two descriptions is provided by the conserved quantities, such as the charges and the energy of the configuration under analysis. These quantities can be easily extracted from the supergravity solution by looking at the large distance decay of the relevant fields; for instance the energy is obtained from the time component of the metric $g_{00}$ and, in the D-brane case, the charge from the RR fields. In the microscopic description, where D-branes are defined as the place where open strings can end, these conserved quantities are obtained from 1-point CFT correlators where the string world-sheet has the topology of the disk [4].

More recently [5, 6], it was showed for 1/4-BPS configurations in type II supergravity that this connection between classical solutions and disk CFT correlators holds also for higher order terms in the large distance expansion, even if they capture dipole instead of conserved charges. In [1], the CFT approach was used to study a class of 1/8-BPS D-brane configurations in type IIB string theory on $R^{1,4} \times S^{1} \times T^{4}$. From the space-time point of view the brane configuration under analysis was a bound state of a D1 and a D5-brane each wrapped $n_{w}$ times on the $S^{1}$ and oscillating in the common $R^{4}$ Dirichlet directions. When this oscillation is described by a purely left (or right) moving null-like wave the system preserves 4 of the original 32 supersymmetries. By studying the disk correlators with the insertion of a closed string vertex corresponding to the massless bosonic degrees of freedom, an asymptotic supergravity solution was derived including dipole and quadrupole terms in the $1 / r$ expansion. In [1] it was also checked, in perturbation theory, that the solution arising from the CFT analysis was indeed 1/8-BPS.

In this paper, we show that the asymptotic solution of [1] is an example of a class of classical configurations which solve the full non-linear equations of supergravity, i.e. the supersymmetry variations with four independent parameters and the equations of motion. The structure of these solutions is similar to that of the configurations studied in [7], and this provided a crucial guidance in the non-linear generalization of the results derived from string correlators. In many respects, the case discussed here can be seen as a direct generalization of the results of [8], but, as suggested by the string analysis, the new supergravity ansatz depends on an additional scalar and vector in the uncompact $R^{1,4}$. Since the original 10D ansatz of [8] could be interpreted as the lift of a $1 / 2$-BPS solution in a 5D ungauged supergravity with two vector multiplets (the STU-model), it is natural to suspect that the generalization discussed here is a solution of an extended 5D supergravity with an extra vector multiplet. We present a 11D lift of the IIB ansatz which makes the embedding of 
this ansatz in such a 5D supergravity manifest (this also provides an alternative and maybe simpler way to check the equations of motions).

In order to provide a concrete solution which falls in the new class discussed in this paper, we use the non-minimal black rings studied in [9], which are solutions of the $\mathcal{N}=2$ supergravity with $n$ vector multiplets. We focus on the case $n=3$, which has one more charge and dipole with the respect to the original black ring of the STU-model [10]. We provide an embedding of the $n=3$ black ring in type IIB string theory, which is a first step towards understanding the ring microstates. In M-theory frame, there does not seem to be any compelling reason to concentrate on solutions with $n=3$ vector multiplets. However, as it emerges from our analysis, this subclass of solutions is very natural from a type IIB perspective because three is the maximum number of vector fields one can turn on while still preserving the $S O(4)$ rotations of the $T^{4}$ directions in the IIB duality frame. It is not obvious to us that the $n=3$ black ring solution can be derived from the one of the STU model by using some generating solution techniques. For instance, U-duality transformations do not seem to be enough to connect the two types of black ring solutions in 10D.

The paper has the following structure. In Section 2 we start from the perturbative solution of [1] and motivate the form of the full-nonlinear solution in a type IIB frame where the charges correspond to momentum, D1 and D5 branes. The solution is given in terms of 4 scalar functions and 5 vectors defined on a hyper-Kahler metric for the $R^{4}$ corresponding to the Dirichlet directions. In Section 3 we provide a Mtheory lift of this class of solutions which makes it manifest how to perform the embedding in a $\mathcal{N}=25 \mathrm{D}$ ungauged supergravity [11], where the scalar manifold is the symmetric space $S O(1,1) \otimes(S O(1,2) / S O(2))$. It is interesting that the lift from type IIB to M-theory used to embed the ansatz of [8] in the $\mathcal{N}=25 \mathrm{D}$ STU-model does not work for the case described in this paper and so we are required to consider a more involved U-duality transformation to get a simple $11 \mathrm{D}$ configuration. In Section 4, we consider a black ring which gives an explicit example of a solution falling in the ansatz discussed. This configuration has a non-trivial horizon area which, as it happened for the original black ring, is given by the quartic invariant of $E_{7}$ [12]. In Section 5 we briefly comment on how to embed the black ring solution under analysis in $\mathrm{AdS}_{3} \times S^{3}$. This is possible by taking a near horizon limit on the solution written in the type IIB duality frame; then one can apply the usual dictionary and use the AdS radius to derive the central charge of the CFT that should describe the microstates of the black ring. In particular, we can choose a ring that has the same charges and dipoles of the perturbative string solution of [1] and starts to differ from it only at the level of quadrupole corrections. In this case the AdS asymptotic is the same as the one of the D1-D5 case and so the dual description should be given by the same D1-D5 CFT describing the StromingerVafa and BMPV black holes. In the spirit of the analysis by Mathur et al. [13], we 
interpret this as evidence for the existence of a class of microstate solutions that have the same dipole terms of the black ring, but then have also quadrupole terms, as predicted by the microscopic D-brane analysis of [1]. In Appendix A we collect the equations of motions, the supersymmetry variations and the duality rules needed to reproduce the results presented in the main text.

\section{A new 1/8-BPS ansatz in type IIB supergravity}

Let us start by recalling the type IIB supergravity solutions belonging to the class discussed in [8]. They are characterised by three charges corresponding to momentum, D1 and D5 branes, and three dipoles related to KK monopoles, D1 and D5 branes. We indicate the $S^{1}$ where all branes are wrapped by the coordinate $y$ and the D5 branes wrap also the $T^{4}$ describing the remaining four compact directions. The uncompact space is topologically $R^{1,4}$, where the $R^{4}$ part is described by a $4 \mathrm{D}$ hyper-Kahler metric $d s_{4}^{2}$. Then the 10D (string frame) metric is given by

$$
\begin{aligned}
d s^{2} & =\frac{1}{\sqrt{Z_{1} Z_{2}}}\left[-\frac{1}{Z_{3}} d \hat{t}^{2}+Z_{3} d \hat{y}^{2}\right]+\sqrt{Z_{1} Z_{2}} d s_{4}^{2}+\sqrt{\frac{Z_{1}}{Z_{2}}} d s_{T^{4}}^{2}, \\
d \hat{t} & =d t+k, \quad d \hat{y}=d y+d t-\frac{d t+k}{Z_{3}}+a_{3},
\end{aligned}
$$

where $Z_{i}$, with $i=1,2,3$, are scalar functions, while $a_{3}$ and $k$ are 1 -forms in $R^{4}$. $d s_{T^{4}}^{2}$ denotes the metric on $T^{4}$, for which we simply take $d s_{T^{4}}^{2}=\sum_{i=1}^{4} d z_{i}^{2}$. The other non-trivial fields are the dilaton and the RR 2-form

$$
C^{(2)}=-\frac{1}{Z_{1}} d \hat{t} \wedge d \hat{y}+a_{1} \wedge\left(d \hat{y}+\frac{d \hat{t}}{Z_{3}}\right)+\gamma_{2}, \quad \mathrm{e}^{2 \phi}=\frac{Z_{1}}{Z_{2}},
$$

where $\gamma_{2}$ is a 2 -form and $a_{1}$ is another 1 -form in $R^{4}$. In order to preserve four supercharges and solve the type IIB supergravity equation of motions, the scalars and the forms appearing in the ansatz above have to satisfy a set of differential equations in $R^{4}$. The 1 -forms $a_{1,3}$ have a self-dual field strength with the respect to the $d s_{4}^{2}$ metric. Also we can define a new form $a_{2}$, with the same property, starting from the self-dual part of the $k$

$$
Z_{2} d a_{2} \equiv d k+*_{4} d k-Z_{1} d a_{1}-Z_{3} d a_{3} .
$$

Thus in summary we have $*_{4} d a_{i}=d a_{i}$ for $i=1,2,3$ and the scalars $Z_{i}$ have to satisfy

$$
d *_{4} d Z_{1}=-d a_{2} \wedge d a_{3}, \quad d *_{4} d Z_{2}=-d a_{1} \wedge d a_{3}, \quad d *_{4} d Z_{3}=-d a_{1} \wedge d a_{2},
$$

Finally the field strength of the 2 -form $\gamma_{2}$ is given in terms of the $Z_{i}$ and $a_{i}$ by

$$
d \gamma_{2}=*_{4} d Z_{2}+a_{1} \wedge d a_{3}
$$


Notice that (2.5) ensures that the r.h.s. of this equation is closed and can be written, at least locally, as the differential of a 2 -form $\gamma$.

The main new feature of the asymptotic solution in [1] is that also the other massless type IIB fields are non-trivial. In particular, at order $1 / r^{3}$ in the large distance expansion, the NS-NS B-field components $B_{t i}$ and $B_{y i}$, where $i$ is in the uncompact $R^{4}$, are non-zero. Then at order $1 / r^{4}$ also the $10 \mathrm{D}$ axion, the $B_{t y}, B_{i j}$ components and the 5 -form RR field strength are non-trivial. It is interesting that, in the string results, the two new $1 / r^{3}$ terms are fixed by the same $R^{4}$ vector, while all the new $1 / r^{4}$ terms are determined by a single scalar. By following the structure of the ansatz summarised above, we will refer to the new scalar and the new vector with self-dual field strength as $Z_{4}$ and $a_{4}$ respectively. Even if the supergravity asymptotic solution arising from string correlators looks very complicated, as many components are non-trivial, the new terms are actually rather similar to those appearing in a class of solutions discussed in [7]. These are 1/4-BPS solutions with only D1 and D5 brane charges which were derived by a U-duality from the solution describing a fundamental string with a left moving wave whose plane of oscillation has one direction in the compact space $T^{4}$. In particular, we are interested in the case where, in the D1/D5 frame, the solution still preserves the $S O(4)$ rotations of the $T^{4}$ directions in the IIB duality frame. This configuration was analysed from the string point of view in [5]. In the 1/4-BPS case all the fields that in our 1/8-BPS case are determined by $Z_{4}$ appear at order $1 / r^{3}$. In the notation of [5], these fields were determined by the function $\mathcal{A}$. The natural guess is that our new scalar $Z_{4}$ should play in the $1 / 8$-BPS case under consideration exactly the same role of $\mathcal{A}$. This provides a guide to complete the non-linear dependence on $Z_{4}$ of the new solution. So we introduce the combination

$$
\alpha=\left(1-\frac{Z_{4}^{2}}{Z_{1} Z_{2}}\right)^{-1}
$$

which should play the same role as the ratio $\tilde{H}_{1} / H_{1}$ in the $1 / 4$-BPS solution of [7]. The other natural guess is that the self-duality property of $d a_{4}$ holds exactly including all the non-linear correction of the 4D hyper-Kahler metric.

Thus by combining the previous supergravity analysis and the string theory results of [1], we are led to the following ansatz

$$
\begin{aligned}
d s^{2} & =\frac{\alpha}{\sqrt{Z_{1} Z_{2}}}\left[-\frac{1}{Z_{3}} d \hat{t}^{2}+Z_{3} d \hat{y}^{2}\right]+\sqrt{Z_{1} Z_{2}} d s_{4}^{2}+\sqrt{\frac{Z_{1}}{Z_{2}}} d s_{T^{4}}^{2}, \\
d \hat{t} & =d t+k, \quad d \hat{y}=d y+d t-\frac{d t+k}{Z_{3}}+a_{3}, \\
\mathrm{e}^{2 \phi} & =\alpha \frac{Z_{1}}{Z_{2}}, \quad C^{(0)}=\frac{Z_{4}}{Z_{1}}, \\
C^{(2)} & =-\frac{\alpha}{Z_{1}} d \hat{t} \wedge d \hat{y}+a_{1} \wedge\left(d \hat{y}+\frac{d \hat{t}}{Z_{3}}\right)+\gamma_{2},
\end{aligned}
$$




$$
\begin{aligned}
& B^{(2)}=-\frac{\alpha Z_{4}}{Z_{1} Z_{2}} d \hat{t} \wedge d \hat{y}+a_{4} \wedge\left(d \hat{y}+\frac{d \hat{t}}{Z_{3}}\right)+\delta_{2}, \\
& F^{(5)}=d\left(\frac{Z_{4}}{Z_{2}}\right) \wedge d z^{4}+\alpha \frac{Z_{2}}{Z_{1}} *_{4} d\left(\frac{Z_{4}}{Z_{2}}\right) \wedge d \hat{t} \wedge d \hat{y} .
\end{aligned}
$$

We denote by $d z^{4}$ the volume form of $T^{4}: d z^{4}=d z_{1} \wedge \ldots \wedge d z_{4}$. The 2-form $\delta_{2}$ plays the same role as the $\gamma_{2}$ in $(2.3)$

$$
d \delta_{2}=*_{4} d Z_{4}+a_{4} \wedge d a_{3}
$$

One can check that the ansatz (2.8) preserves four supercharges and solves the type IIB equations of motion, provided that the definition of $a_{2}$ in (2.4) is slightly modified

$$
Z_{2} d a_{2} \equiv d k+*_{4} d k-Z_{1} d a_{1}-Z_{3} d a_{3}+2 Z_{4} d a_{4}
$$

and the equation of the scalars take the form

$$
\begin{array}{ll}
d *_{4} d Z_{1}=-d a_{2} \wedge d a_{3}, & d *_{4} d Z_{2}=-d a_{1} \wedge d a_{3}, \\
d *_{4} d Z_{3}=-d a_{1} \wedge d a_{2}+d a_{4} \wedge d a_{4}, & d *_{4} d Z_{4}=-d a_{3} \wedge d a_{4} .
\end{array}
$$

We will see that Eqs. (2.10) and (2.11) implies the equations of motions of a $\mathcal{N}=2$ $5 \mathrm{D}$ ungauged supergravity with three vector multiplets for the case static solutions.

\section{M-theory lift and $\mathcal{N}=2$ truncation}

The standard approach to lift (2.1)-(2.3) to M-theory is to take three T-dualities, one along the $S^{1}$ direction $y$ where the D-branes are wrapped, and the remaining two in the $T^{4}$. By trying the same approach starting from (2.8), we get a rather complicated $11 \mathrm{D}$ solution which we could not truncate to any 5D supergravity. The main problem is that the new 1 -form $a_{4}$ in the $B$-field (2.8) appears in the 11D metric and is not on the same footing as the other forms which appear in the potential $A^{(3)}$. This suggests that the solution to this problem is to look for a different duality chain. We indicate with $S$ a type IIB S-duality, with $T_{a b}$ a pair of T-dualities along the Cartesian coordinates $z_{a}, z_{b}, a, b=1, \ldots, 4$ of the (square) $T^{4}$, and $T_{y}$ a T-duality along the $S^{1}$ parametrised by $y$. Then the chain of dualities we are going to consider is

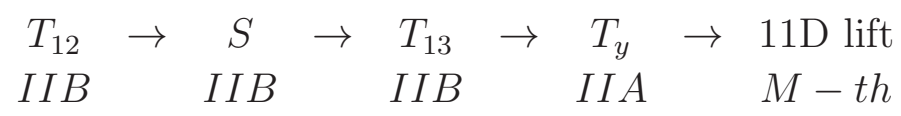

It is not difficult to follow the fate of the 1-forms $a_{I}$ in (2.8) and realise that these dualities bring the type IIB ansatz to a nice M-theory frame, where all $a_{I}$ appear as components the 3 -form potential $A^{(3)}$. By using the rules summarised in the Appendix A, we can follow how the ansatz (2.8) transforms under the duality chain 
in (3.1). In order to carry out this computation, it is convenient to use (A.2) and derive the potential $C^{(4)}$ for the self-dual field strength in (2.8)

$$
C^{(4)}=\frac{Z_{4}}{Z_{2}} d z^{4}-\frac{\alpha Z_{4}}{Z_{1} Z_{2}} \gamma_{2} \wedge d \hat{t} \wedge d \hat{y}+x_{3} \wedge\left(d \hat{y}+\frac{d \hat{t}}{Z_{3}}\right)
$$

where

$$
d x_{3}=d a_{4} \wedge \gamma_{2}-a_{1} \wedge\left(d \delta_{2}-a_{4} \wedge d a_{3}\right) .
$$

It is also convenient to work in a 'democratic' formalism where both the R-R field strengths and their Hodge dual are explicitly written in the solution. Clearly the high-degree $n$-forms, with $n>5$, contain redundant information, but after some T-dualities they can contribute to a low degree form with $n \leq 5$. For our specific case only $C^{(6)}$ is needed; it is defined via (A.4) and (A.2) and it is given by

$$
C^{(6)}=\left[-\frac{\alpha}{Z_{2}} d \hat{t} \wedge d \hat{y}+a_{2} \wedge\left(d \hat{y}+\frac{d \hat{t}}{Z_{3}}\right)+\gamma_{1}\right] \wedge d z^{4}-\frac{\alpha Z_{4}}{Z_{1} Z_{2}} \gamma_{2} \wedge \gamma_{2} \wedge d \hat{t} \wedge d \hat{y}
$$

with

$$
d \gamma_{1}=*_{4} d Z_{1}+a_{2} \wedge d a_{3}
$$

The result for the $11 \mathrm{D}$ metric is

$$
\begin{aligned}
d s_{11}^{2} & =-\left(\frac{\alpha}{Z_{1} Z_{2} Z_{3}}\right)^{2 / 3} d \hat{t}^{2}+\left(\frac{Z_{1} Z_{2} Z_{3}}{\alpha}\right)^{1 / 3} d s_{4}^{2} \\
& +\alpha^{2 / 3}\left(Z_{1} Z_{2} Z_{3}\right)^{1 / 3}\left[\frac{d w_{1} d \bar{w}_{1}}{Z_{1}}+\frac{d w_{2} d \bar{w}_{2}}{Z_{2}}+\frac{d w_{3} d \bar{w}_{3}}{\alpha Z_{3}}\right. \\
& \left.+\frac{Z_{4}}{Z_{1} Z_{2}}\left(d w_{1} d \bar{w}_{2}+d w_{2} d \bar{w}_{1}\right)\right]
\end{aligned}
$$

where the complex coordinates $w_{1,2}$ parametrise the $T^{4}$ that was already present in the type IIB setup, while $w_{3}$ parametrises an extra $T^{2}$ obtained by combining the $y$ direction together with the M-theory circle $z$

$$
w_{1} \equiv z_{2}-\mathrm{i} z_{3}, \quad w_{2} \equiv z_{1}+\mathrm{i} z_{4}, \quad w_{3} \equiv y+\mathrm{i} z
$$

Notice that the volume of the $T^{6}$ defined by the second and third line of (3.6) is independent of all functions $Z_{I}$ and is simply equal to one. For the 11D 3-form potential we obtain

$$
\begin{aligned}
A^{(3)} & =\left(-\frac{\alpha d \hat{t}}{Z_{1}}+a_{1}\right) \wedge \frac{d w_{1} \wedge d \bar{w}_{1}}{-2 \mathrm{i}}+\left(-\frac{\alpha d \hat{t}}{Z_{2}}+a_{2}\right) \wedge \frac{d w_{2} \wedge d \bar{w}_{2}}{-2 \mathrm{i}} \\
& +\left(-\frac{d \hat{t}}{Z_{3}}+a_{3}\right) \wedge \frac{d w_{3} \wedge d \bar{w}_{3}}{-2 \mathrm{i}}+\left(-\frac{\alpha Z_{4}}{Z_{1} Z_{2}} d \hat{t}+a_{4}\right) \wedge \frac{d w_{1} \wedge d \bar{w}_{2}+d w_{2} \wedge d \bar{w}_{1}}{-2 \mathrm{i}}
\end{aligned}
$$

The non-trivial components in (3.6) and (3.8) are the only ones that are invariant under the two continuous transformations

$$
\left(w_{1} \rightarrow \mathrm{e}^{\mathrm{i} \theta} w_{1}, w_{2} \rightarrow \mathrm{e}^{\mathrm{i} \theta} w_{2}\right) \quad \text { and } \quad w_{3} \rightarrow \mathrm{e}^{\mathrm{i} \phi} w_{3},
$$


and the discrete transformation

$$
\left(A^{(3)} \rightarrow-A^{(3)}, w_{1} \leftrightarrow \bar{w}_{1}, w_{2} \leftrightarrow \bar{w}_{2}, w_{3} \leftrightarrow \bar{w}_{3}\right) .
$$

These are symmetries of the original 11D supergravity and thus the restriction to the fields that are invariant under (3.9) and (3.10) provides a consistent truncation. The result (3.8) for $A^{(3)}$ suggests to introduce

$$
J_{i}=\frac{d w_{i} \wedge d \bar{w}_{i}}{-2 \mathrm{i}}, \quad i=1,2,3 \quad \text { and } \quad J_{4}=\frac{d w_{1} \wedge d \bar{w}_{2}+d w_{2} \wedge d \bar{w}_{1}}{-2 \mathrm{i}} .
$$

These are $(1,1)$-forms with the respect to the complex structure (3.7). So we can follow [14] and list the $\mathcal{N}=2$ vector multiplets obtained by reducing the $11 \mathrm{D}$ ansatz on $T^{6}$ down to 5D. The intersection numbers of the $J_{I}$ will play an important role

$$
J_{I} \wedge J_{J} \wedge J_{K}=\frac{1}{6} C_{I J K} J \wedge J \wedge J
$$

where the Kahler form $J$ is given in (3.14). The $C$ 's are clearly fully symmetric under the exchange of their indices and, in our case, $C_{123}$ and all its permutations are equal to 1 , while the only non-trivial coupling involving $I=4$ is $C_{344}$

$$
C_{123}=1, \quad C_{344}=-2 .
$$

The scalars of the vector multiplets can be read by decomposing $J$ along the $J_{I}$

$$
J=\mathrm{i} h_{i \bar{k}} d w^{i} \wedge d \bar{w}^{k}=\sum_{I} t^{I} J_{I}, \quad \text { with } \quad d s_{T_{6}}^{2}=h_{i \bar{k}} d w^{i} d \bar{w}^{k} .
$$

In writing the Kahler form $J$ for our case we keep only the components that are non-trivial in (3.6) and obtain

$$
J=\alpha^{2 / 3}\left(Z_{1} Z_{2} Z_{3}\right)^{1 / 3}\left[\frac{1}{Z_{1}} J_{1}+\frac{1}{Z_{2}} J_{2}+\frac{1}{\alpha Z_{3}} J_{3}+\frac{Z_{4}}{Z_{1} Z_{2}} J_{4}\right] .
$$

This yields the moduli coordinate $t^{I}$. Since, as noticed above, the total volume of the compact space is just one in our case the $t^{I}$ automatically satisfy the relation $t^{I} t^{J} t^{K} C_{I J K} / 6=1$. By using (3.13) for the intersection numbers for our $J_{I}$ in (3.11), we can write the constraint on the moduli coordinates as

$$
\mathcal{V}=\frac{1}{6} t^{I} t^{J} t^{K} C_{I J K}=t^{1} t^{2} t^{3}-t^{3}\left(t^{4}\right)^{2}=1
$$

This constraint defines the symmetric space $S O(1,1) \otimes(S O(1,2) / S O(2))$, which appears in one of the possible $\mathcal{N}=25 \mathrm{D}$ truncation of the maximal ungauged supergravity.

In a similar way we can decompose $A^{(3)}$ along the $J_{I}$ and read the vectors $A_{I}$ obtained in the reduction $A^{(3)}=\sum_{I} A_{I} J_{I}$. In our case we have

$$
A^{(3)}=\sum_{c=1}^{2}\left(-\frac{\alpha d \hat{t}}{Z_{c}}+a_{c}\right) J_{c}+\left(-\frac{d \hat{t}}{Z_{3}}+a_{3}\right) J_{3}+\left(-\frac{\alpha Z_{4}}{Z_{1} Z_{2}} d \hat{t}+a_{4}\right) J_{4} .
$$


Contrary to what happens in the expansion of the Kahler form (3.15), in the case of $A^{(3)}$ there is no constraint relating the components $A_{c}$. This means that the number of the $5 \mathrm{D}$ vectors is equal to the number $h_{11}$ of $(1,1)$-forms, while the number of independent scalars obtained from $J$ is just $h_{11}-1$. This field content fits in $h_{11}-1$ $\mathcal{N}=2$ vector multiplets, while the additional vector in (3.17) is part of the graviton multiplet.

In our case (3.11) $h_{11}=4$, so we have three vector multiplets. The explicit form of the intersection numbers (3.13) fixes completely the 5D action

$$
\int d^{5} x\left[\sqrt{\left|g_{5}\right|} R-\frac{Q_{I J}}{4} *_{5} F^{I} \wedge F^{J}-\frac{Q_{I J}}{2} *_{5} d t^{I} \wedge d t^{J}-\frac{C_{I J K}}{24} F^{I} \wedge F^{J} \wedge A^{K}\right]
$$

where the quadratic form $Q$ in the kinetic terms is

$$
Q_{I J}=-\left.\frac{\partial}{\partial t^{I}} \frac{\partial}{\partial t^{J}} \log \mathcal{V}\right|_{\mathcal{V}=1}
$$

and $F^{I}=d A^{I}$. With the identifications (3.14) and (3.17) between the fields in the $\mathcal{N}=2$ supergravity, one can check that the 5D equations of motions are implied by the self-duality property $*_{4} d a_{I}=d a_{I}$ together with Eqs. (2.10), (2.11). For instance the equations of motion for the vectors are

$$
d\left(Q_{I J} *_{5} F^{J}\right)=\frac{C_{I J K}}{2} F^{J} \wedge F^{K},
$$

and its magnetic components are equivalent to (2.11). Thus we can see the solutions falling in the ansatz (3.6) and (3.8) as the 11D lift of $5 \mathrm{D}$ solutions of an $\mathcal{N}=2$ supergravity.

\section{A black ring solution}

In (2.8) the NS-NS and the R-R 2-forms have a very similar structure, where the only qualitative difference is that $\delta_{2}$ is related to the scalar $Z_{4}$ appearing the $d t \wedge d y$ term of $B^{(2)}$, while the corresponding components for $C^{(2)}$ are completely independent. So this ansatz allows for solutions with equal, but non-trivial, charges for a fundamental string and a NS5-brane. The simplest possible example of a solution of this type is probably a black hole characterised by four parameters $Q_{I}$ determining its charges. It is natural to expect that there exists also a class of black ring solutions with four parameters fixing the charges and four parameters related to dipoles. Such a black ring solution has indeed already appeared as a particular case of solutions in [9], that constructed black ring solutions in $5 \mathrm{D} \mathcal{N}=2$ supergravity coupled to an arbitrary number of vector multiplets. Here we emphasize the 11D interpretation 
of this solution and, more importantly, the fact that it is U-dual to a IIB solution belonging to the ansatz that emerges from the string theory computation of [1]. We believe that this black ring solution cannot be derived by using some generating solution techniques from the black ring with three charges and three dipoles.

Following the by now well established construction of BPS solutions with two axial symmetries, we choose the $4 \mathrm{D}$ part $d s_{4}^{2}$ in (2.8) to be simply the flat Euclidean metric and rewrite it in the Gibbons-Hawking form:

$$
d s_{4}^{2}=V^{-1}(d \tau+A)^{2}+V d s_{3}^{2}, \quad *_{3} d A=d V,
$$

where $\tau$ is a linear combination of the two axial isometries of two orthogonal planes in $R^{4}$ and $d s_{3}^{2}$ is the flat 3 -dimensional metric

$$
d s_{3}^{2}=d r^{2}+r^{2} d \theta^{2}+r^{2} \sin ^{2} \theta d \phi^{2} .
$$

Since we are focusing on a flat 4D metric, the Gibbons-Hawking potentials are

$$
V=\frac{1}{r}, \quad A=\cos \theta d \phi .
$$

For configurations preserving a $U(1) \times U(1)$ symmetry, such as the black rings, we can express $k$, the $a_{I}$ 's and the $Z_{I}$ 's of the ansatz (2.8) in terms of harmonic functions $K_{I}, L_{I}$ and $M$ on $R^{3}$

$$
\begin{aligned}
& a_{I}=\frac{K_{I}}{V}(d \tau+A)+\bar{a}_{I}, \quad *_{3} d \bar{a}_{I}=-d K_{I}, \quad(I=1,2,3,4) \\
& Z_{1}=L_{1}+\frac{K_{2} K_{3}}{V}, \quad Z_{2}=L_{2}+\frac{K_{1} K_{3}}{V} \\
& Z_{3}=L_{3}+\frac{K_{1} K_{2}}{V}-\frac{K_{4}^{2}}{V}, \quad Z_{4}=L_{4}+\frac{K_{3} K_{4}}{V} \\
& k=\left(M+\frac{\sum_{i=1}^{3} L_{i} K_{i}-2 L_{4} K_{4}}{2 V}+\frac{\left(K_{1} K_{2}-K_{4}^{2}\right) K_{3}}{V^{2}}\right)(d \tau+A)+\omega \\
& *_{3} d \omega=V d M-M d V+\frac{1}{2} \sum_{i=1}^{3}\left(d L_{i} K_{i}-L_{i} d K_{i}\right)-\left(d L_{4} K_{4}-L_{4} d K_{4}\right) .
\end{aligned}
$$

The harmonic functions that generate black ring solutions carrying 4 charges and 4 dipole charges are centred at a distance $R$ from the origin of the 3D space (4.2). The coordinates from this special point will be indicated with $\Sigma$ and $\theta_{\Sigma}$

$$
\Sigma=\sqrt{r^{2}+R^{2}-2 R r \cos \theta}, \quad \cos \theta_{\Sigma}=\frac{r \cos \theta-R}{\Sigma} .
$$

The most general allowed harmonic functions that describe a ring wrapping the fiber direction $\tau$ in (4.1) are

$$
K_{I}=\frac{d_{I}}{\Sigma}, \quad L_{I}=\ell_{I}+\frac{Q_{I}}{\Sigma} \quad(I=1, \ldots, 4), \quad M=m_{0}+\frac{m}{\Sigma} .
$$


The parameters appearing in these harmonic functions should be chosen so as to avoid Dirac-Misner singularities in the 1-form on $R^{3}, \omega$. By focusing in a neighbourhood of the point $r=0$ this requires

$$
m_{0}=-\frac{m}{R},
$$

while regularity around $\Sigma=0$ implies

$$
m=\frac{R}{2}\left(\sum_{i=i}^{3} \ell_{i} d_{i}-2 \ell_{4} d_{4}\right) .
$$

Note that condition (4.7) also guarantees that the coefficient of $(d \tau+A)$ in (4.4) (which we call $\mu$ ) vanishes at the origin of polar coordinates $r=0$, and hence that $k$ is regular as a 1 -form on $R^{4}$.

In order to insure the absence of closed time-like curves, the $Z_{I}$ and the coefficient of $d \tau^{2}$ should be positive. The conditions on $Z_{I}$ are obviously satisfied if we take $\ell_{i}$, $Q_{i}$ and $d_{i}$, with $i=1,2,3$, positive and $d_{1} d_{2}-d_{4}^{2}>0$. Further conditions that are sufficient to insure the positivity of $\alpha$ are

$$
\begin{aligned}
& \ell_{1} \ell_{2}-\ell_{4}^{2}>0, \quad \ell_{2} Q_{1}+\ell_{1} Q_{2}-2 \ell_{4} Q_{4} \geq 0, \quad \ell_{1} d_{1}+\ell_{2} d_{2}-2 \ell_{4} d_{4} \geq 0 \\
& d_{1} Q_{1}+d_{2} Q_{2}-2 d_{4} Q_{4} \geq 0, \quad Q_{1} Q_{2}-Q_{4}^{2} \geq 0
\end{aligned}
$$

To analyze the condition on the $d \tau^{2}$ coefficient it is convenient to rewrite the $5 \mathrm{D}$ metric in the first line of (3.6)

$$
d s_{E}^{2}=-\left(\frac{\alpha}{Z_{1} Z_{2} Z_{3}}\right)^{2 / 3}(d t+k)^{2}+\left(\frac{\alpha}{Z_{1} Z_{2} Z_{3}}\right)^{-1 / 3} d s_{4}^{2},
$$

by completing squares with respect to $d \tau$. Then we obtain

$$
\begin{aligned}
d s_{E}^{2}= & \left(\frac{\alpha}{Z_{1} Z_{2} Z_{3}}\right)^{2 / 3} \frac{\tilde{I}_{4}}{V^{2}}\left[d \tau+A-\frac{\mu V^{2}}{\tilde{I}_{4}}(d t+\omega)\right]^{2} \\
& +\left(\frac{\alpha}{Z_{1} Z_{2} Z_{3}}\right)^{-1 / 3} \frac{V}{\tilde{I}_{4}}\left[\tilde{I}_{4} d s_{3}^{2}-(d t+\omega)^{2}\right]
\end{aligned}
$$

where

$$
\begin{aligned}
\tilde{I}_{4}= & \alpha^{-1} Z_{1} Z_{2} Z_{3} V^{2}-\mu^{2} V^{2} \\
= & \frac{1}{2} \sum_{I<J=1}^{3} K_{I} K_{J} L_{I} L_{J}-\frac{1}{4} \sum_{I=1}^{3} K_{I}^{2} L_{I}^{2}+V\left(L_{1} L_{2}-L_{4}^{2}\right) L_{3} \\
& +\left(K_{1} L_{1}+K_{2} L_{2}-K_{3} L_{3}\right) K_{4} L_{4}-K_{4}^{2} L_{1} L_{2}-K_{1} K_{2} L_{4}^{2} \\
& -2 M\left(K_{1} K_{2}-K_{4}^{2}\right) K_{3}-M V\left(\sum_{I=1}^{3} K_{I} L_{I}-2 K_{4} L_{4}\right)-M^{2} V^{2} .
\end{aligned}
$$


Absence of closed time-like curves requires that $\tilde{I}_{4}$ be everywhere positive. Then around $\Sigma=0$ we must have

$$
\tilde{I}_{4} \approx \frac{\tilde{J}_{4}}{\Sigma^{4}}>0
$$

where

$$
\begin{aligned}
\tilde{J}_{4}= & \frac{1}{2} \sum_{I<J=1}^{3} d_{I} d_{J} Q_{I} Q_{J}-\frac{1}{4} \sum_{I=1}^{3} d_{I}^{2} Q_{I}^{2} \\
& +\left(d_{1} Q_{1}+d_{2} Q_{2}-d_{3} Q_{3}\right) d_{4} Q_{4}-d_{4}^{2} Q_{1} Q_{2}-d_{1} d_{2} Q_{4}^{2} \\
& -2 m\left(d_{1} d_{2}-d_{4}^{2}\right) d_{3},
\end{aligned}
$$

and thus one needs $\tilde{J}_{4}>0$.

We will now show that the geometry has a regular horizon of finite area at $\Sigma=0$. The behavior of the metric functions for $\Sigma \rightarrow 0$ is

$$
\begin{aligned}
& Z_{1} \approx \frac{R d_{2} d_{3}}{\Sigma^{2}}, \quad Z_{2} \approx \frac{R d_{1} d_{3}}{\Sigma^{2}}, \quad Z_{3} \approx \frac{R\left(d_{1} d_{2}-d_{4}^{2}\right)}{\Sigma^{2}}, \\
& Z_{4} \approx \frac{R d_{3} d_{4}}{\Sigma^{2}}, \quad \alpha^{-1} \approx \frac{d_{1} d_{2}-d_{4}^{2}}{d_{1} d_{2}}, \\
& \mu \approx \frac{R^{2}\left(d_{1} d_{2}-d_{4}^{2}\right) d_{3}}{\Sigma^{3}}, \quad \omega=O(\Sigma), \quad \tilde{I}_{4} \approx \frac{\tilde{J}_{4}}{\Sigma^{4}} .
\end{aligned}
$$

Looking at the metric in M-theory frame, one sees that the $T^{6}$ part of the metric is finite, as far as $d_{1} d_{2} \neq d_{4}^{2}$. The gauge fields $A_{I}(I=1, \ldots, 4)$ have a potential divergence proportional to $d \tau+A$; the divergent terms however cancel, thanks to the identities

$$
\begin{aligned}
& -\frac{\alpha \mu}{Z_{1}}+\frac{K_{1}}{V}=O\left(\Sigma^{0}\right), \quad-\frac{\alpha \mu}{Z_{2}}+\frac{K_{2}}{V}=O\left(\Sigma^{0}\right), \\
& -\frac{\mu}{Z_{3}}+\frac{K_{3}}{V}=O\left(\Sigma^{0}\right),
\end{aligned}
$$

The term $\left(\frac{\alpha}{Z_{1} Z_{2} Z_{3}}\right)^{2 / 3} \frac{\tilde{I}_{4}}{V^{2}}$ that appears in $d s_{E}^{2}$ is finite and the term $\frac{\mu V^{2}}{\tilde{I}_{4}}$ goes to zero. Finally the determinant of the horizon metric (i.e. of the submanifold $t=$ const, $\Sigma=0)$ is

$$
\operatorname{det} g_{\text {Hor }}=\Sigma^{2}\left(\tilde{I}_{4} \Sigma^{2} \sin ^{2} \theta_{\Sigma}-\omega_{\phi}^{2}\right) \approx \tilde{J}_{4} \sin ^{2} \theta_{\Sigma}
$$

so that the area of the horizon is proportional to $\tilde{J}_{4}^{1 / 2}$.

With the standard 5D-4D map [15], we can use the isometry along $\tau$ and reduce the black ring solution (4.6) to a four dimensional black hole. So we expect that the combination $\tilde{I}_{4}$ can be expressed in terms of the quartic invariant $\mathcal{I}$ of the symmetry group $\mathrm{E}_{7}$. This invariant can be expressed in terms of the matrices

$$
Z_{A B}=\sum_{\hat{g}, h=1}^{8}\left(x_{\hat{g} h}+i y_{\hat{g} \hat{h}}\right)\left(\gamma^{\hat{g} \hat{h}}\right)_{A B}, \quad Z^{A B}=\sum_{\hat{g}, \hat{h}=1}^{8}\left(x_{\hat{g} \hat{h}}+i y_{\hat{g} \hat{h}}\right)\left(\gamma^{\hat{g} \hat{h}}\right)^{A B},
$$


where the $\gamma^{g}$ 's are the $8 \times 8$ blocks of the $S O(8)$ Gamma matrices with chiral indices $A, B=1, \ldots, 8$. Then, by following [12], the quartic invariant can be written as

$$
\mathcal{J}=\frac{1}{256}\left[Z_{A B} Z^{B C} Z_{C D} Z^{D A}-\frac{1}{4}\left(Z_{A B} Z^{B A}\right)^{2}+\operatorname{Pf}\left(Z_{A B}\right)+\operatorname{Pf}\left(Z^{A B}\right)\right]
$$

where Pf indicates the Pfaffian of $Z$. As suggested by the expression for the cycles related to our ansatz (3.11), one can use the dictionary (4.20) and check that the general expression for the quartic invariant (4.19) agrees with the result in (4.12)

$$
\begin{aligned}
& x_{12} \rightarrow Q_{1}, \quad x_{34} \rightarrow Q_{2}, \quad x_{56} \rightarrow Q_{3}, \quad x_{13} \rightarrow Q_{4}, \quad x_{24} \rightarrow Q_{4}, \\
& y_{12} \rightarrow d_{1}, y_{34} \rightarrow d_{2}, \quad y_{56} \rightarrow d_{3}, \quad y_{13} \rightarrow-d_{4}, y_{24} \rightarrow-d_{4}, \quad y_{78} \rightarrow-2 m .
\end{aligned}
$$

The $5 \mathrm{D}$ mass is proportional to

$$
M=\ell_{2} \ell_{3} \widetilde{Q}_{1}+\ell_{1} \ell_{3} \widetilde{Q}_{2}+\left(\ell_{1} \ell_{2}-\ell_{4}^{2}\right) \widetilde{Q}_{3}-2 \ell_{3} \ell_{4} \widetilde{Q}_{4},
$$

where $\widetilde{Q}_{I}(I=1, \ldots, 4)$ are the four charges carried by the solution:

$$
\widetilde{Q}_{1}=Q_{1}+d_{2} d_{3}, \quad \widetilde{Q}_{2}=Q_{2}+d_{1} d_{3}, \quad \widetilde{Q}_{3}=Q_{3}+d_{1} d_{2}-d_{4}^{2}, \quad \widetilde{Q}_{4}=Q_{4}+d_{3} d_{4} .
$$

In the IIB duality frame $\widetilde{Q}_{1}, \widetilde{Q}_{2}, \widetilde{Q}_{3}$ correspond, respectively, to D1, D5 and momentum charges; moreover there is an equal amount of F1 and NS5 charge, given by $\widetilde{Q}_{4}$.

The angular momenta with respect to the 5D Cartan angles $\tilde{\psi}$ and $\tilde{\phi}$ (defined as $\tau=\tilde{\psi}+\tilde{\phi}, \phi=\tilde{\psi}-\tilde{\phi})$ are given by

$$
\begin{aligned}
& J_{\tilde{\psi}}=\frac{1}{2}\left(\sum_{i=1}^{3} d_{i} \tilde{Q}_{i}-2 d_{4} \tilde{Q}_{4}\right)-\frac{1}{2}\left(d_{1} d_{2}-d_{4}^{2}\right) d_{3}+R\left(\sum_{i=1}^{3} \ell_{i} d_{i}-2 \ell_{4} d_{4}\right) \\
& J_{\tilde{\phi}}=\frac{1}{2}\left(\sum_{i=1}^{3} d_{i} \tilde{Q}_{i}-2 d_{4} \tilde{Q}_{4}\right)-\frac{1}{2}\left(d_{1} d_{2}-d_{4}^{2}\right) d_{3} .
\end{aligned}
$$

\section{$5 \quad$ Discussion and open problems}

We found an exact supersymmetric solution of type IIB supergravity comprising all the fields that are sourced by the class of D1, D5 and P bound states studied in [1]. The ansatz we find is more general than the one that was previously used to describe three-charge bound state geometries [8] and that, when reduced to $5 \mathrm{D}$, is equivalent to the STU model (i.e. $\mathcal{N}=25 \mathrm{D}$ supergravity with two vector multiplets). We show that a suitable chain of dualities relates our ansatz to the M-theory lift of $\mathcal{N}=25$ D supergravity with three vector multiplets. 
We have also examined a black ring solution in this extended ansatz, carrying four charges and four dipole charges. Though this solution is already contained in the general class of solutions of 5D supergravity constructed in [9], an explicit 11D embedding of this black ring solution is, as far as we know, new. Moreover, we relate this M-theory solution to a duality-equivalent IIB solution. Knowing the solution in type IIB frame allows one to investigate the existence of 'near-horizon' limits containing an $A d S_{3}$ factor, and hence to attempt a microscopic interpretation of the solution. As explained for example in [16], the near-horizon solution is obtained by taking

$$
r \sim \alpha^{\prime 2}, \quad R \sim \alpha^{\prime 2}, \quad Q_{1,2,4} \sim \alpha^{\prime}, \quad Q_{3} \sim \alpha^{\prime 2}, \quad d_{1,2,4} \sim \alpha^{\prime}, \quad d_{3} \sim 1
$$

and by sending $\alpha^{\prime} \rightarrow 0$. This amounts to dropping the constants $\ell_{I}$ from $L_{1}, L_{2}$ and $L_{4}$, but not from $L_{3}$. To obtain the asymptotic limit of the near-horizon solution one then sends $r \equiv \frac{\rho^{2}}{4} \rightarrow \infty$ and is left with the geometry:

$$
d s^{2}=\frac{\bar{\alpha} \rho^{2}}{4 \sqrt{\tilde{Q}_{1} \tilde{Q}_{2}}}\left(-d t^{2}+d y^{2}\right)+4 \sqrt{\tilde{Q}_{1} \tilde{Q}_{2}} \frac{d \rho^{2}}{\rho^{2}}+4 \sqrt{\tilde{Q}_{1} \tilde{Q}_{2}} d \Omega_{3}^{2}+\sqrt{\frac{\tilde{Q}_{1}}{\tilde{Q}_{2}}} d s_{T^{4}}^{2}
$$

with

$$
\bar{\alpha}=\left(1-\frac{\tilde{Q}_{4}^{2}}{\tilde{Q}_{1} \tilde{Q}_{2}}\right)^{-1}
$$

This is $A d S_{3} \times S^{3} \times T^{4}$, where the radius of $A d S_{3}$ is $\ell_{A d S}=2\left(\tilde{Q}_{1} \tilde{Q}_{2}\right)^{1 / 4}$. The dilaton goes to the constant value

$$
\mathrm{e}^{2 \phi}=\bar{\alpha} \frac{\tilde{Q}_{1}}{\tilde{Q}_{2}}
$$

and thus, the usual computation gives for the central charge

$$
c=\frac{3 \ell_{A d S}}{2 G_{3}}=\frac{6 V_{4}}{\pi^{4} g^{2} \alpha^{\prime 4}} \tilde{Q}_{1} \tilde{Q}_{2} \bar{\alpha}^{-1},
$$

where $V_{4}$ is the volume of $T^{4}$. To attempt a microscopic interpretation one should express $c$ in terms of the integer charge numbers; for the STU charges, this is done for example in [16] (note that the charges $Q_{I}$ in [16] are 4 times $^{1}$ our $\tilde{Q}_{I}$ ):

$$
\tilde{Q}_{1}=\frac{4 \pi^{4} g \alpha^{\prime 3}}{V_{4}} N_{D 1}, \quad \tilde{Q}_{2}=\frac{g \alpha^{\prime}}{4} N_{D 5}, \quad \tilde{Q}_{3}=\frac{4 \pi^{4} g^{2} \alpha^{\prime 4}}{V_{4} R_{y}^{2}} N_{P},
$$

where $R_{y}$ is the radius of the $y$ circle and $N_{D 1}, N_{D 5}, N_{P}$ are the numbers of D1branes, D5-branes and quantized units of momentum along $y$. The quantity $\tilde{Q}_{4}$ represents both the charge of fundamental strings wrapped on $y$ and of NS5-branes wrapped on $y$ and $T^{4}$, and hence it is quantized as

$$
\tilde{Q}_{4}=\frac{4 \pi^{4} g^{2} \alpha^{\prime 3}}{V_{4}} N_{F 1}=\frac{\alpha^{\prime}}{4} N_{N S 5}
$$

\footnotetext{
${ }^{1}$ This is due to the factor of 4 between the $3 \mathrm{D}$ radial coordinate $r$ and the $4 \mathrm{D}$ one $\rho$.
} 
Note that this imposes the constraint that the moduli combination $\frac{V_{4}}{(2 \pi)^{4} g^{2} \alpha^{\prime 2}}$ be a rational number. When we substitute these relations in (5.5), we find

$$
c=6\left(N_{D 1} N_{D 5}-N_{F 1} N_{N S 5}\right) .
$$

The CFT with central charge (5.8) that is dual to our general black ring is not known, and it would be interesting to further investigate its properties. One can however look at the subset of black ring solutions with $\tilde{Q}_{4}=N_{F 1}=N_{N S 5}=0$ (but $d_{4} \neq 0$ ): it follows from our analysis that these are new black ring solutions that should be describable within the same D1-D5 CFT, with central charge $c=6 N_{D 1} N_{D 5}$, that describes the Strominger-Vafa and the BMPV black holes. A consistent amount of work $[16,17]$ has been devoted to identifying the subset of states of the D1-D5 CFT that correspond to the STU model black rings, but a completely satisfactory understanding of this problem is still lacking. We point out here that the same CFT should also contain a subset of states that are dual to our more general class of black ring solutions: characterizing these states and reproducing the entropy given in (4.14) for $\tilde{Q}_{4}=0$ by counting them remains an interesting open problem. Moreover in [18] a new supersymmetric phase of the D1-D5 CFT that has no known gravity dual was found: it would be interesting to see if our generalized black rings could be relevant in that context.

\section{Acknowledgements}

We thank I. Bena, G. Dall'Agata, S. El-Showk, S. Ferrara, M. Graña, S. Mathur, J.F. Morales, M. Shigemori, H. Triendl, D. Turton, B. Vercnocke and N. Warner for several enlightening discussions. $\mathrm{RR}$ has been partially supported by STFC Standard Grant ST/J000469/1 "String Theory, Gauge Theory and Duality".

\section{A Supergravity conventions}

\section{A.1 IIB}

The IIB action in string frame is

$$
\begin{aligned}
\Gamma= & \int \mathrm{e}^{-2 \phi}\left(\sqrt{-g} R+4 * d \phi \wedge d \phi-\frac{1}{2} * H^{(3)} \wedge H^{(3)}\right)-\frac{1}{2} * F^{(1)} \wedge F^{(1)} \\
& -\frac{1}{2} * F^{(3)} \wedge F^{(3)}-\frac{1}{4} * F^{(5)} \wedge F^{(5)}+\frac{1}{2} H^{(3)} \wedge F^{(3)} \wedge C^{(4)}
\end{aligned}
$$

where

$$
H^{(3)}=d B^{(2)}, \quad F^{(p+1)}=d C^{(p)}-H^{(3)} \wedge C^{(p-2)},
$$


and we define the Hodge dual in $d$ dimensions as

$$
*\left(d x^{i_{1}} \wedge \ldots \wedge d x^{i_{p}}\right)=\sqrt{-g} d x^{i_{p+1}} \wedge \ldots \wedge d x^{i_{d}} \epsilon_{i_{p+1} \ldots i_{d}}^{i_{1} \ldots i_{p}}
$$

with the orientation $\epsilon_{t y x_{1} x_{2} x_{3} x_{4} z_{1} z_{2} z_{3} z_{4}}=1$. The 5 -forms $F^{(5)}$ is taken to be self-dual.

We use the same supersymmetry conventions as in appendix A of [1].

To perform T-dualities we find convenient to work in the democratic formalism, and hence introduce the field strengths $F^{(7)}$ and $F^{(9)}$, dual to $F^{(3)}$ and $F^{(1)}$, as

$$
F^{(7)}=-* F^{(3)}, \quad F^{(9)}=* F^{(1)} .
$$

The corresponding gauge potentials $C^{(6)}$ and $C^{(8)}$ are related to the field strengths by (A.2).

The S-duality symmetry of IIB acts as

$$
\begin{aligned}
& d s^{2} \rightarrow \mathrm{e}^{-\phi} d s^{2}, \quad \phi \rightarrow-\phi, \quad C^{(0)} \rightarrow C^{(0)}, \quad B^{(2)} \rightarrow C^{(2)}, \quad C^{(2)} \rightarrow-B^{(2)}, \\
& C^{(4)} \rightarrow C^{(4)}-B^{(2)} \wedge C^{(2)} .
\end{aligned}
$$

Note that this transformation leaves invariant the Einstein metric $e^{-\phi / 2} d s^{2}$ and the 5-form $F^{(5)}$.

\section{A.2 T-duality}

Denote by $y$ the direction along which one performs T-duality and by $x^{\mu}$ the remaining coordinates. It is convenient to write the string metric, B-field and gauge fields as

$$
\begin{aligned}
d s^{2} & =G_{y y}\left(d y+A_{\mu} d x^{\mu}\right)^{2}+\hat{g}_{\mu \nu} d x^{\mu} d x^{\nu}, \\
B^{(2)} & =B_{\mu y} d x^{\mu} \wedge\left(d y+A_{\mu} d x^{\mu}\right)+\hat{B}^{(2)}, \\
C^{(p)} & =C_{y}^{(p-1)} \wedge\left(d y+A_{\mu} d x^{\mu}\right)+\hat{C}^{(p)},
\end{aligned}
$$

where the forms $\hat{B}^{(2)}, C_{y}^{(p-1)}$ and $\hat{F}^{(p)}$ are along the $x^{\mu}$ directions.

The T-duality transformed fields are

$$
\begin{aligned}
d \tilde{s}^{2} & =G_{y y}^{-1}\left(d y-B_{\mu y} d x^{\mu}\right)^{2}+\hat{g}_{\mu \nu} d x^{\mu} d x^{\nu}, \quad e^{2 \tilde{\Phi}}=\frac{e^{2 \Phi}}{G_{y y}} \\
\tilde{B}^{(2)} & =-A_{\mu} d x^{\mu} d y+\hat{B}^{(2)} \\
\tilde{C}^{(p)} & =\hat{C}^{(p-1)} \wedge\left(d y-B_{\mu y} d x^{\mu}\right)+C_{y}^{(p)} .
\end{aligned}
$$




\section{A.3 M-theory}

The M-theory action is

$$
\Gamma_{11}=\int \sqrt{-g_{11}} R_{11}-\frac{1}{2} *_{11} d A^{(3)} \wedge d A^{(3)}+\frac{1}{6} A^{(3)} \wedge d A^{(3)} \wedge d A^{(3)},
$$

where $R_{11}$ and $*_{11}$ denote the Ricci scalar and Hodge dual with respect to the $11 \mathrm{D}$ metric $g_{11}$. We use the orientation $\epsilon_{t x_{1} x_{2} x_{3} x_{4} z_{1} z_{2} z_{3} z_{4} y z}=1$.

\section{References}

[1] S. Giusto, R. Russo, and D. Turton, "New D1-D5-P geometries from string amplitudes," JHEP 1111 (2011) 062, arXiv:1108.6331 [hep-th].

[2] M. Duff, R. R. Khuri, and J. Lu, "String solitons,"

Phys.Rept. 259 (1995) 213-326, arXiv:hep-th/9412184 [hep-th].

[3] J. Polchinski, S. Chaudhuri, and C. V. Johnson, "Notes on D-branes," arXiv:hep-th/9602052 [hep-th].

[4] P. Di Vecchia, M. Frau, I. Pesando, S. Sciuto, A. Lerda, and R. Russo, "Classical p-branes from boundary state," Nucl. Phys. B507 (1997) 259-276, arXiv:hep-th/9707068.

[5] S. Giusto, J. F. Morales, and R. Russo, "D1D5 microstate geometries from string amplitudes," JHEP 03 (2010) 130, arXiv:0912.2270 [hep-th].

[6] W. Black, R. Russo, and D. Turton, "The supergravity fields for a D-brane with a travelling wave from string amplitudes,"

Phys. Lett. B694 (2010) 246-251, arXiv:1007.2856 [hep-th].

[7] I. Kanitscheider, K. Skenderis, and M. Taylor, "Fuzzballs with internal excitations," JHEP 06 (2007) 056, arXiv:0704.0690 [hep-th].

[8] I. Bena and N. P. Warner, "One ring to rule them all ... and in the darkness bind them?," Adv.Theor.Math.Phys. 9 (2005) 667-701, arXiv:hep-th/0408106 [hep-th];

I. Bena and N. P. Warner, "Bubbling supertubes and foaming black holes," Phys.Rev. D74 (2006) 066001, arXiv:hep-th/0505166 [hep-th];

P. Berglund, E. G. Gimon, and T. S. Levi, "Supergravity microstates for BPS black holes and black rings," JHEP 0606 (2006) 007, arXiv:hep-th/0505167 [hep-th]. 
[9] H. Elvang, R. Emparan, D. Mateos, and H. S. Reall, "Supersymmetric black rings and three-charge supertubes," Phys.Rev. D71 (2005) 024033,

arXiv:hep-th/0408120 [hep-th];

J. P. Gauntlett and J. B. Gutowski, "General concentric black rings,"

Phys.Rev. D71 (2005) 045002, arXiv:hep-th/0408122 [hep-th].

[10] H. Elvang, R. Emparan, D. Mateos, and H. S. Reall, "A Supersymmetric black ring," Phys.Rev.Lett. 93 (2004) 211302, arXiv:hep-th/0407065 [hep-th].

[11] M. Gunaydin, G. Sierra, and P. Townsend, "The Geometry of N=2

Maxwell-Einstein Supergravity and Jordan Algebras,"

Nucl.Phys. B242 (1984) 244.

[12] E. Cremmer and B. Julia, "The SO(8) Supergravity," Nucl.Phys. B159 (1979) 141.

[13] S. D. Mathur, "The fuzzball proposal for black holes: An elementary review," Fortsch. Phys. 53 (2005) 793-827, arXiv: hep-th/0502050;

I. Bena and N. P. Warner, "Black holes, black rings and their microstates,"

Lect. Notes Phys. 755 (2008) 1-92, arXiv: hep-th/0701216; K. Skenderis and M. Taylor, "The fuzzball proposal for black holes,"

Phys. Rept. 467 (2008) 117-171, arXiv:0804.0552 [hep-th];

V. Balasubramanian, J. de Boer, S. El-Showk, and I. Messamah, "Black Holes as Effective Geometries," Class.Quant.Grav. 25 (2008) 214004, arXiv:0811.0263 [hep-th]; B. D. Chowdhury and A. Virmani, "Modave Lectures on Fuzzballs and Emission from the D1-D5 System," arXiv:1001.1444 [Unknown].

[14] A. Cadavid, A. Ceresole, R. D'Auria, and S. Ferrara, "Eleven-dimensional supergravity compactified on Calabi-Yau threefolds,"

Phys.Lett. B357 (1995) 76-80, arXiv:hep-th/9506144 [hep-th];

G. Papadopoulos and P. Townsend, "Compactification of D = 11 supergravity on spaces of exceptional holonomy," Phys.Lett. B357 (1995) 300-306, arXiv:hep-th/9506150 [hep-th].

[15] D. Gaiotto, A. Strominger, and X. Yin, "New connections between 4-D and 5-D black holes," JHEP 0602 (2006) 024, arXiv:hep-th/0503217 [hep-th]; D. Gaiotto, A. Strominger, and X. Yin, "5D black rings and 4D black holes," JHEP 0602 (2006) 023, arXiv:hep-th/0504126 [hep-th] .

[16] I. Bena and P. Kraus, "Microscopic description of black rings in AdS/CFT," JHEP 12 (2004) 070, arXiv:hep-th/0408186.

[17] N. Iizuka and M. Shigemori, "A Note on D1-D5-J system and 5-D small black 
ring," JHEP 0508 (2005) 100, arXiv:hep-th/0506215 [hep-th];

A. Dabholkar, N. Iizuka, A. Iqubal, and M. Shigemori, "Precision microstate counting of small black rings," Phys.Rev.Lett. 96 (2006) 071601, arXiv:hep-th/0511120 [hep-th];

L. F. Alday, J. de Boer, and I. Messamah, "What is the dual of a dipole?," Nucl.Phys. B746 (2006) 29-57, arXiv:hep-th/0511246 [hep-th];

A. Dabholkar, N. Iizuka, A. Iqubal, A. Sen, and M. Shigemori, "Spinning strings as small black rings," JHEP 0704 (2007) 017, arXiv:hep-th/0611166 [hep-th].

[18] I. Bena, B. D. Chowdhury, J. de Boer, S. El-Showk, and M. Shigemori, "Moulting Black Holes," arXiv:1108.0411 [hep-th]. 